International Journal of Pure and Applied Mathematics

Volume 107 No. 2 2016, 343-356

ISSN: 1311-8080 (printed version); ISSN: 1314-3395 (on-line version)

url: http://www.ijpam.eu

doi: 10.12732/ijpam.v107i2.5

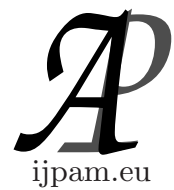

\title{
COMMON FIXED POINTS OF $R$-WEAKLY \\ COMMUTING MAPPINGS AND ITS VARIANTS FOR MULTIPLICATIVE EXPANSIVE MAPPINGS
}

\author{
Poonam Nagpal ${ }^{1}$, Sudhir Kumar Garg ${ }^{2}$, Sanjay Kumar ${ }^{3}$, Shin Min Kang ${ }^{4} \S$ \\ 1,2,3 Department of Mathematics \\ Deenbandhu Chhotu Ram University of Science and Technology \\ Murthal, Sonipat, 131039, Haryana, INDIA \\ ${ }^{4}$ Department of Mathematics and RINS \\ Gyeongsang National University \\ Jinju, 52828, KOREA
}

\begin{abstract}
In this paper, we introduce the notions of R-weakly commuting and its variants for multiplicative expansive mappings in multiplicative metric spaces and prove common fixed point theorems for these mappings. Moreover, we prove a common fixed point theorem for weakly reciprocally continuous mappings. We also provide examples in support of our results.
\end{abstract}

AMS Subject Classification: $47 \mathrm{H} 10,54 \mathrm{H} 25$

Key Words: multiplicative metric spaces, multiplicative expansive mappings, R-weakly commuting mappings, $\mathbf{R}$-weakly commuting mappings of types, weakly reciprocally continuous mappings

\section{Introduction and Preliminaries}

It is well know that the set of positive real numbers $\mathbb{R}_{+}$is not complete according to the usual metric. To overcome this problem, in 2008, Bashirov et al. [2] introduced the concept of multiplicative metric spaces as follows:

\footnotetext{
Received: December 8, 2015

(c) 2016 Academic Publications, Ltd.

Published: April 5, 2016

url: www.acadpubl.eu
}

${ }^{\S}$ Correspondence author 
Definition 1.1. Let $X$ be a nonempty set. A multiplicative metric is a mapping $d: X \times X \rightarrow \mathbb{R}_{+}$satisfying the following conditions:

(i) $d(x, y) \geq 1$ for all $x, y \in X$ and $d(x, y)=1$ if and only if $x=y$;

(ii) $d(x, y)=d(y, x)$ for all $x, y \in X$;

(iii) $d(x, y) \leq d(x, z) \cdot d(z, y)$ for all $x, y, z \in X$ (multiplicative triangle inequality).

Then the mapping $d$ together with $X$, that is, $(X, d)$ is a multiplicative metric space.

Example 1.2. ([6]) Let $\mathbb{R}_{+}^{n}$ be the collection of all $n$-tuples of positive real numbers. Let $d^{*}: \mathbb{R}_{+}^{n} \times \mathbb{R}_{+}^{n} \rightarrow \mathbb{R}$ be defined as follows:

$$
d^{*}(x, y)=\left|\frac{x_{1}}{y_{1}}\right|^{*} \cdot\left|\frac{x_{2}}{y_{2}}\right|^{*} \ldots\left|\frac{x_{n}}{y_{n}}\right|^{*},
$$

where $x=\left(x_{1}, \ldots, x_{n}\right), y=\left(y_{1}, \ldots, y_{n}\right) \in \mathbb{R}_{+}^{n}$ and $|\cdot|^{*}: \mathbb{R}_{+} \rightarrow \mathbb{R}_{+}$is defined by

$$
|a|^{*}= \begin{cases}a & \text { if } a \geq 1 \\ \frac{1}{a} & \text { if } a<1\end{cases}
$$

Then it is obvious that all conditions of a multiplicative metric are satisfied. Therefore $\left(\mathbb{R}_{+}^{n}, d\right)$ is a multiplicative metric space.

Example 1.3. ([12]) Let $d: \mathbb{R} \times \mathbb{R} \rightarrow[1, \infty)$ be defined as $d(x, y)=a^{|x-y|}$, where $x, y \in \mathbb{R}$ and $a>1$. Then $d$ is a multiplicative metric and $(\mathbb{R}, d)$ is a multiplicative metric space. We may call it usual multiplicative metric spaces.

Remark 1.4. We note that the Example 1.2 is valid for positive real numbers and Example 1.3 is valid for all real numbers.

Example 1.5. ([12]) Let $(X, d)$ be a metric space. Define a mapping $d_{a}$ on $X$ by

$$
d_{a}(x, y)=a^{d(x, y)}= \begin{cases}1 & \text { if } x=y \\ a & \text { if } x \neq y\end{cases}
$$

where $x, y \in X$ and $a>1$. Then $d_{a}$ is a multiplicative metric and $\left(X, d_{a}\right)$ is known as the discrete multiplicative metric space.

Example 1.6. ([1]) Let $X=C^{*}[a, b]$ be the collection of all real-valued multiplicative continuous functions on $[a, b] \subset \mathbb{R}_{+}$. Then $(X, d)$ is a multiplicative metric space with $d$ defined by $d(f, g)=\sup _{x \in[a, b]}\left|\frac{f(x)}{g(x)}\right|$ for arbitrary $f, g \in X$. 
Remark 1.7. ([12]) We note that multiplicative metrics and metric spaces are independent.

Indeed, the mapping $d^{*}$ defined in Example 1.2 is multiplicative metric but not metric as it does not satisfy triangular inequality. Consider

$$
d^{*}\left(\frac{1}{3}, \frac{1}{2}\right)+d^{*}\left(\frac{1}{2}, 3\right)=\frac{3}{2}+6=7.5<9=d^{*}\left(\frac{1}{3}, 3\right) .
$$

On the other hand the usual metric on $\mathbb{R}$ is not multiplicative metric as it doesnt satisfy multiplicative triangular inequality, since

$$
d(2,3) \cdot d(3,6)=3<4=d(2,6) .
$$

One can refer to $[3,6]$ for detailed multiplicative metric topology.

Definition 1.8. Let $(X, d)$ be a multiplicative metric space. Then a sequence $\left\{x_{n}\right\}$ in $X$ said to be

(1) a multiplicative convergent to $x$ if for every multiplicative open ball $B_{\epsilon}(x)=\{y \mid d(x, y)<\epsilon\}, \epsilon>1$, there exists $N \in \mathbb{N}$ such that $x_{n} \in B_{\epsilon}(x)$ for all $n \geq N$, that is, $d\left(x_{n}, x\right) \rightarrow 1$ as $n \rightarrow \infty$.

(2) a multiplicative Cauchy sequence if for all $\epsilon>1$, there exists $N \in \mathbb{N}$ such that $d\left(x_{n}, x_{m}\right)<\epsilon$ for all $m, n>N$, that is, $d\left(x_{n}, x_{m}\right) \rightarrow 1$ as $n, m \rightarrow \infty$.

(3) We call a multiplicative metric space complete if every multiplicative Cauchy sequence in it is multiplicative convergent to $x \in X$.

Remark 1.9. The set of positive real numbers $\mathbb{R}_{+}$is not complete according to the usual metric. Let $X=\mathbb{R}_{+}$and the sequence $\left\{x_{n}\right\}=\left\{\frac{1}{n}\right\}$. It is obvious $\left\{x_{n}\right\}$ is a multiplicative Cauchy sequence in $X$ with respect to usual metric and $X$ is not a complete metric space, since $0 \notin \mathbb{R}_{+}$. In case of a multiplicative metric space, we take a sequence $\left\{x_{n}\right\}=\left\{a^{\frac{1}{n}}\right\}$, where $a>1$. Then $\left\{x_{n}\right\}$ is a multiplicative Cauchy sequence since for $n \geq m$,

$$
\begin{aligned}
d\left(x_{n}, x_{m}\right) & =\left|\frac{x_{n}}{x_{m}}\right|=\left|\frac{a^{\frac{1}{n}}}{a^{\frac{1}{m}}}\right|=\left|a^{\frac{1}{n}-\frac{1}{m}}\right| \\
& \leq a^{\frac{1}{m}-\frac{1}{n}}<a^{\frac{1}{m}}<\epsilon \quad \text { if } m>\frac{\log a}{\log \epsilon}
\end{aligned}
$$

where $|a|=\left\{\begin{array}{ll}a & \text { if } a \geq 1, \\ \frac{1}{a} & \text { if } a<1 .\end{array}\right.$ Also, $\left\{x_{n}\right\} \rightarrow 1$ as $n \rightarrow \infty$ and $1 \in \mathbb{R}_{+}$. Hence $(X, d)$ is a complete multiplicative metric space. 
In 2012, Özavsar and Çevikel [6] gave the concept of multiplicative contraction mappings and proved some fixed point theorems of such mappings in a multiplicative metric space.

Definition 1.10. Let $f$ be a mapping of a multiplicative metric space $(X, d)$ into itself. Then $f$ is said to be a multiplicative contraction if there exists a real constant $\lambda \in[0,1)$ such that

$$
d(f x, f y) \leq d^{\lambda}(x, y) \quad \text { for all } x, y \in X .
$$

Also they proved the Banach Contraction Principle in the setting of multiplicative metric spaces as follows:

Theorem 1.11. Let $f$ be a multiplicative contraction mapping of a complete multiplicative metric space $(X, d)$ into itself. Then $f$ has a unique fixed point.

In 1984, Wang et al. [13] and in 1993 Rhoades [11] proved some fixed point theorems for expansion mappings, which corresponds to some contractive mappings in metric spaces.

Now we define expansive mappings in the setting of multiplicative metric spaces as follows:

Definition 1.12. Let $f$ be a mapping of a multiplicative metric space $(X, d)$ into itself. Then $f$ is said to be a multiplicative expansive mapping if there exists a constant $\alpha>1$ such that for all $x, y \in X$, we have

$$
d(f x, f y) \geq d^{\alpha}(x, y) .
$$

Example 1.13. Let $X=\mathbb{R}_{+}$with usual multiplicative metric $d$. Define $f: X \rightarrow X$ by

$$
f x= \begin{cases}\frac{1}{x^{2}} & \text { if } x \neq 1 \\ 1 & \text { if } x=1\end{cases}
$$

Then clearly,

$$
d(f x, f y) \geq d^{\alpha}(x, y)
$$

holds for $1<\alpha \leq 2$, which implies that $f$ is a multiplicative expansive mapping.

\section{2. $R$-Weakly Commuting Mappings and its Variants}

In 1994, Pant [7] introduced the $R$-weakly commuting mappings in metric spaces. 
In 1997, Pathak et al. [10] improved the notion of $R$-weakly commuting mappings to $R$-weakly commuting mappings of type $\left(A_{g}\right)$ and $R$-weakly commuting mappings of type $\left(A_{f}\right)$ in metric spaces.

In 2009, Kumar and Garg [5] introduced the concept of $R$-weakly commuting mappings of type $(P)$ in metric spaces

Now in similar mode we introduce the notions of $R$-weakly commuting mappings and its variants in multiplicative metric spaces as follows:

Definition 2.1. Let $f$ and $g$ be two mappings of a multiplicative metric space $(X, d)$ into itself. Then $f$ and $g$ are said to be

(1) $R$-weakly commuting if there exists some $R>0$ such that $d(f g x, g f x) \leq$ $d^{R}(f x, g x)$ for all $x \in X$.

(2) $R$-weakly commuting of type $\left(A_{f}\right)$ if there exists some $R>0$ such that $d(f g x, g g x) \leq d^{R}(f x, g x)$ for all $x \in X$.

(3) $R$-weakly commuting of type $\left(A_{g}\right)$ if there exists some $R>0$ such that $d(g f x, f f x) \leq d^{R}(f x, g x)$ for all $x \in X$.

(4) $R$-weakly commuting of type $(P)$ if there exists some $R>0$ such that $d(f f x, g g x) \leq d^{R}(f x, g x)$ for all $x \in X$.

Example 2.2. Let $X=(2, \infty)$. Define $d: X \times X \rightarrow \mathbb{R}_{+}$by $d(x, y)=\left|\frac{x}{y}\right|$. Then $(X, d)$ is a multiplicative metric space. Define $f, g: X \rightarrow X$ by $f x=x$ and $g x=x-1$ for all $x \in X$. Then

$$
\begin{aligned}
& d(f x, g x)=d(x, x-1)=\frac{x}{x-1}, \\
& d(f g x, g f x)=1, \quad d(f g x, g g x)=\frac{x-1}{x-2}, \\
& d(f f x, g f x)=\frac{x}{x-1} \quad \text { and } \quad d(f f x, g g x)=\frac{x}{x-2} .
\end{aligned}
$$

Then clearly

$$
d(f g x, g f x) \leq d^{R}(f x, g x)
$$

for all $x \in X$ and for some $R>0$, which implies that $f$ and $g$ are $R$-weakly commuting mappings. Clearly $f$ and $g$ are also $R$-weakly commuting mappings of type $\left(A_{g}\right)$.

Example 2.3. Let $X=\mathbb{R}_{+}$. Define $d: X \times X \rightarrow \mathbb{R}_{+}$by $d(x, y)=\left|\frac{x}{y}\right|$. Then $(X, d)$ is a multiplicative metric space. Define $f, g: X \rightarrow X$ by $f x=x^{2}$ and $g x=x$ for all $x \in X$. Then

$$
d(f x, g x)=d\left(x^{2}, x\right)=|x|, \quad d(f g x, g g x)=d\left(x^{2}, x\right)=|x| .
$$


Then clearly

$$
d(f g x, g g x) \leq d^{R}(f x, g x)
$$

for all $x \in X$ and for some $R>0$, therefore, $f$ and $g$ are $R$-weakly commuting of type $\left(A_{f}\right)$.

Example 2.4. Let $X=\mathbb{R}_{+}$. Define $d: X \times X \rightarrow \mathbb{R}_{+}$by $d(x, y)=\left|\frac{x}{y}\right|$. Then $(X, d)$ is a multiplicative metric space. Define $f, g: X \rightarrow X$ by $f x=3 x$ and $g x=x^{2}$ for all $x \in X$. Then

$$
d(f x, g x)=d\left(3 x, x^{2}\right)=\left|\frac{3}{x}\right|, \quad d(f f x, g g x)=d\left(9 x, x^{2}\right)=\left|\frac{9}{x^{2}}\right| .
$$

Then clearly

$$
d(f f x, g g x) \leq d^{R}(f x, g x)
$$

for all $x \in X$ and for some $R>2>0$, which implies that $f$ and $g$ are $R$-weakly commuting of type $(P)$.

Remark 2.5. We have suitable examples to show that $R$-weakly commuting mappings, $R$-weakly commuting of type $\left(A_{f}\right), R$-weakly commuting of type $\left(A_{g}\right)$ and $R$-weakly commuting of type $(P)$ are distinct.

Example 2.6. Let $X=[-2,2]$. Define $d: X \times X \rightarrow \mathbb{R}_{+}$by $d(x, y)=$ $a^{|x-y|}$, where $a>1$. Then $(X, d)$ is a multiplicative metric space. Define $f, g$ : $X \rightarrow X$ by $f x=|x|$ and $g x=|x|-2$ for all $x \in X$. Then

$$
\begin{aligned}
& d(f x, g x)=a^{2}, \quad d(f g x, g f x)=a^{2(2-|x|)}, \quad d(g f x, f f x)=a^{2}, \\
& d(f g x, g g x)=a^{2}, \quad \text { and } \quad d(f f x, g g x)=a^{2|x|}
\end{aligned}
$$

for all $x \in X$. Hence we have

(1) For $R=2, f$ and $g$ are $R$-weakly commuting, $R$-weakly commuting of type $\left(A_{f}\right), R$-weakly commuting of type $\left(A_{g}\right)$ and $R$-weakly commuting of type $(P)$.

(2) For $R=\frac{3}{2}, f$ and $g$ are $R$-weakly commuting of type $\left(A_{f}\right), R$-weakly commuting of type $\left(A_{g}\right)$, but $f$ and $g$ are not $R$-weakly commuting of type $(P)$ and $R$-weakly commuting.

Example 2.7. Let $X=\mathbb{R}$. Define $d: X \times X \rightarrow \mathbb{R}_{+}$by $d(x, y)=a^{|x-y|}$, where $a>1$. Then $(X, d)$ is a multiplicative metric space. Define $f, g: X \rightarrow X$ by $f x=x$ and $g x=2 x-1$ for all $x \in X$. Then

$$
\begin{aligned}
& d(f x, g x)=a^{|x-1|}, \quad d(f g x, g f x)=1, \quad d(g f x, f f x)=a^{|x-1|}, \\
& d(f g x, g g x)=a^{2|x-1|} \quad \text { and } \quad d(f f x, g g x)=a^{3|x-1|}
\end{aligned}
$$


for all $x \in X$. Hence we have

(1) $f$ and $g$ are $R$-weakly commuting for all $\mathbb{R}_{+}$.

(2) For $R=3, f$ and $g$ are $R$-weakly commuting of type $\left(A_{f}\right), R$-weakly commuting of type $\left(A_{g}\right)$ and $R$-weakly commuting of type $(P)$.

(3) For $R=2, f$ and $g$ are $R$-weakly commuting of type $\left(A_{f}\right), R$-weakly commuting of type $\left(A_{g}\right)$, but $f$ and $g$ are not $R$-weakly commuting of type $(P)$.

Example 2.8. Let $X=[0,1]$. Define $d: X \times X \rightarrow \mathbb{R}_{+}$by $d(x, y)=a^{|x-y|}$, where $a>1$. Then $(X, d)$ be a multiplicative metric space. Define $f, g: X \rightarrow X$ by $f x=x$ and $g x=x^{2}$ for all $x \in X$. Then

$$
\begin{aligned}
& d(f x, g x)=a^{|x(x-1)|}=a^{x(1-x)}, \\
& d(f g x, g f x)=1, \quad d(g f x, f f x)=a^{x(1-x)}, \\
& d(f g x, g g x)=a^{x^{2}(1+x)(1-x)} \quad \text { and } \quad d(f f x, g g x)=a^{x(1-x)\left(x^{2}+x+1\right)}
\end{aligned}
$$

for all $x \in X$. Hence we have

(1) $f$ and $g$ are $R$-weakly commuting for all $\mathbb{R}_{+}$.

(2) For $0<R<1, f$ and $g$ are not $R$-weakly commuting of type $\left(A_{f}\right)$, $R$-weakly commuting of type $\left(A_{g}\right)$ and $R$-weakly commuting of type $(P)$.

(3) For $1<R<2, f$ and $g$ are $R$-weakly commuting of type $\left(A_{g}\right)$, but $f$ and $g$ are not $R$-weakly commuting of type $\left(A_{f}\right)$ and $R$-weakly commuting of type $(P)$.

(4) For $2<R<3, f$ and $g$ are $R$-weakly commuting of type $\left(A_{g}\right)$ and $R$-weakly commuting of type $\left(A_{f}\right)$, but $f$ and $g$ are not $R$-weakly commuting of type $(P)$.

(5) For $R>3, f$ and $g$ are $R$-weakly commuting of type $\left(A_{g}\right), R$-weakly commuting of type $\left(A_{f}\right)$ and $R$-weakly commuting of type $(P)$.

Theorem 2.9. Let $f$ and $g$ be $R$-weakly commuting mappings of a complete multiplicative metric space $(X, d)$ into itself satisfying the conditions

$$
\begin{gathered}
g(X) \subset f(X), \\
d(f x, f y) \geq d^{q}(g x, g y)
\end{gathered}
$$

for all $x, y \in X$, where $q>1$.

Assume that $f$ is continuous. Then $f$ and $g$ have a unique common fixed point.

Proof. Let $x_{0} \in X$. Since $g(X) \subset f(X)$, there exists $x_{1} \in X$ such that $f x_{1}=g x_{0}$. In general, there exists $x_{n+1} \in X$ such that

$$
y_{n}=f x_{n+1}=g x_{n} .
$$


From $\left(C_{2}\right)$, consider

$$
\begin{aligned}
d\left(y_{n}, y_{n+1}\right) & =d\left(g x_{n}, g x_{n+1}\right) \leq d^{\frac{1}{q}}\left(f x_{n}, f x_{n+1}\right) \\
& \leq d^{\frac{1}{q}}\left(g x_{n-1}, g x_{n}\right) \leq d^{\frac{1}{q^{2}}}\left(f x_{n-1}, f x_{n}\right) \\
& =d^{\frac{1}{q^{L}}}\left(y_{n-2}, y_{n-1}\right) .
\end{aligned}
$$

In general, we have

$$
d\left(y_{n}, y_{n+1}\right) \leq d^{k^{n}}\left(y_{0}, y_{1}\right)
$$

where $k=\frac{1}{q}<1$.

Now for $m, n \in \mathbb{N}$ with $n<m$, consider

$$
\begin{aligned}
d\left(y_{n}, y_{m}\right) & \leq d\left(y_{n}, y_{n+1}\right) \cdot d\left(y_{n+1}, y_{n+2}\right) \cdots d\left(y_{m-1}, y_{m}\right) \\
& \leq d^{k^{n}}\left(y_{0}, y_{1}\right) \cdot d^{k^{n+1}}\left(y_{0}, y_{1}\right) \cdots d^{k^{m-1}}\left(y_{0}, y_{1}\right) \\
& \leq d^{\frac{k^{n}}{1-k}}\left(y_{0}, y_{1}\right) \\
& \rightarrow 1
\end{aligned}
$$

as $n \rightarrow \infty$. It follows that the sequence $\left\{y_{n}\right\}$ is a multiplicative Cauchy sequence. Since $(X, d)$ is complete, we have

$$
\lim _{n \rightarrow \infty} f x_{n}=\lim _{n \rightarrow \infty} g x_{n}=z .
$$

Since $f$ and $g$ are $R$-weakly commuting mappings, so there exists some $R>1$ such that

$$
d(f g x, g f x) \leq d^{R}(f x, g x)
$$

for all $x \in X$ and hence

$$
d\left(f g x_{n}, g f x_{n}\right) \leq d^{R}\left(f x_{n}, g x_{n}\right) .
$$

Letting $n \rightarrow \infty$ and using continuity of $f$, we have

$$
\lim _{n \rightarrow \infty} f g x_{n}=\lim _{n \rightarrow \infty} g f x_{n}=f z .
$$

Consider

$$
d\left(g f x_{n}, g z\right) \leq d^{\frac{1}{q}}\left(f f x_{n}, f z\right) .
$$

As $n \rightarrow \infty$, we get $\lim _{n \rightarrow \infty} g f x_{n}=g z=f z$.

Now to show $z$ is a fixed point of $f$ and $g$. 
Again consider

$$
d\left(g z, g x_{n}\right) \leq d^{\frac{1}{q}}\left(f z, f x_{n}\right)
$$

Letting $n \rightarrow \infty$, we get

$$
d(g z, z) \leq d^{\frac{1}{q}}(f z, z)
$$

which implies that $f z=z$. Hence $z$ is a fixed point of $f$ and $g$.

Uniqueness follows easily from $\left(C_{2}\right)$.

Example 2.10. Let $X=\mathbb{R}_{+}$with the usual multiplicative metric $d$. Define $f$ and $g: X \rightarrow X$ by $f x=x^{2}$ and $g x=x$ for all $x \in X$. Then $d(f g x, g f x)=1$ and $d(f x, g x)=\left|\frac{1}{x}\right|$. Then clearly

$$
d(f g x, g f x) \leq d^{R}(f x, g x)
$$

for $R>0$, that is, $f$ and $g$ are $R$-weakly commuting. Also $f$ and $g$ satisfy the condition

$$
d(f x, f y) \geq d^{q}(g x, g y)
$$

where $1<q<2$. Clearly $f$ is continuous and $g(X)=f(X)=X$, that is, $g(X) \subset f(X)$. All the conditions of Theorem 2.9 hold. Hence $f$ and $g$ have a unique common fixed point 1.

\section{Weakly Reciprocally Continuous Mappings}

In 1998, Pant [8] introduced a new notion of continuity, known as reciprocal continuity and, in 1999, Pant [9] generalized the notion of reciprocal continuity to weak reciprocal continuity in a metric space.

Now we define this concepts in a multiplicative metric space as follows:

Definition 3.1. Let $f$ and $g$ be two mappings of a multiplicative metric space $(X, d)$ into itself. Then $f$ and $g$ are said to be

(1) reciprocally continuous if $\lim _{n \rightarrow \infty} f g x_{n}=f t$ and $\lim _{n \rightarrow \infty} g f x_{n}=g t$, whenever $\left\{x_{n}\right\}$ is a sequence in $X$ such that $\lim _{n \rightarrow \infty} f x_{n}=\lim _{n \rightarrow \infty} g x_{n}=t$ for some $t \in X$.

(2) weakly reciprocally continuous if $\lim _{n \rightarrow \infty} f g x_{n}=f t$ or $\lim _{n \rightarrow \infty} g f x_{n}=g t$, whenever $\left\{x_{n}\right\}$ is a sequence in $X$ such that $\lim _{n \rightarrow \infty} f x_{n}=\lim _{n \rightarrow \infty} g x_{n}=t$ for some $t \in X$.

Recently Kang et al. [4] introduced the notion of compatible mappings. 
Definition 3.2. Let $f$ and $g$ be two mappings of a multiplicative metric space $(X, d)$ into itself. Then $f$ and $g$ are said to be compatible if $\lim _{n \rightarrow \infty} d\left(f g x_{n}\right.$, $\left.g f x_{n}\right)=1$, whenever $\left\{x_{n}\right\}$ is a sequence in $X$ such that $\lim _{n \rightarrow \infty} f x_{n}=\lim _{n \rightarrow \infty} g x_{n}=t$ for some $t \in X$.

Proposition 3.3. Let $f$ and $g$ be compatible mappings of a multiplicative metric space $(X, d)$ into itself. If $f t=g t$ for some $t \in X$, then $f g t=f f t=$ $g g t=g f t$.

Theorem 3.4. Let $f$ and $g$ be weakly reciprocally continuous mappings of a complete multiplicative metric space $(X, d)$ into itself satisfying the conditions $\left(C_{1}\right)$ ans $\left(C_{2}\right)$.

Assume that $f$ and $g$ are either compatible or $R$-weakly commuting of type $\left(A_{g}\right)$ or $R$-weakly commuting of type $\left(A_{f}\right)$ or $R$-weakly commuting of type $(P)$. Then $f$ and $g$ have a unique common fixed point.

Proof. From the proofs of Theorem 2.9, the sequence $\left\{y_{n}\right\}$ is a multiplicative Cauchy sequence. fore

Since $(X, d)$ is complete, there exists $z \in X$ such that $\lim _{n \rightarrow \infty} y_{n}=z$. There-

$$
\lim _{n \rightarrow \infty} f x_{n+1}=\lim _{n \rightarrow \infty} g x_{n}=z .
$$

Suppose that $f$ and $g$ are compatible mappings. Now as $f$ and $g$ are weakly reciprocally continuous of $f$ and $g$. Therefore, we have $\lim _{n \rightarrow \infty} f g x_{n}=f z$ or $\lim _{n \rightarrow \infty} g f x_{n}=g z$.

Let $\lim _{n \rightarrow \infty} f g x_{n}=f z$. By compatibility of $f$ and $g$, we have

$$
\lim _{n \rightarrow \infty} f g x_{n}=\lim _{n \rightarrow \infty} g f x_{n}=f z .
$$

And also,

$$
\lim _{n \rightarrow \infty} g f x_{n+1}=\lim _{n \rightarrow \infty} g g x_{n}=f z .
$$

Now

$$
d\left(g z, g g x_{n}\right) \leq d^{\frac{1}{q}}\left(f z, f g x_{n}\right)
$$

Taking $n \rightarrow \infty$, we get

$$
d(g z, f z) \leq d^{\frac{1}{q}}(f z, f z)=1
$$

Hence $f z=g z$. Again, since $f$ and $g$ are compatible, by Proposition 3.3, we get $g f z=f g z=f f z=g g z$. Using $\left(C_{2}\right)$, we obtain

$$
d(g z, g g z) \leq d^{\frac{1}{q}}(f z, f g z) \leq d^{\frac{1}{q}}(g z, g g z)
$$


that is, $g z=g g z$. We also have $g z=g g z=f g z$ and $g z$ is a common fixed point of $f$ and $g$.

Next, suppose that $\lim _{n \rightarrow \infty} g f x_{n}=g z$. The assumption $g(X) \subset f(X)$, which implies that $g z=f u$ for some $u \in X$ and hence $\lim _{n \rightarrow \infty} g f x_{n}=f u$.

Now,

$$
\lim _{n \rightarrow \infty} g f x_{n+1}=\lim _{n \rightarrow \infty} g g x_{n}=f u .
$$

Using $(C)$, we get

$$
d\left(g u, g g x_{n}\right) \leq d^{\frac{1}{q}}\left(f u, f g x_{n}\right)
$$

Taking $n \rightarrow \infty$, we get

$$
d(g u, f u) \leq d^{\frac{1}{q}}(f u, f u)=1
$$

Then we get $f u=g u$. Now since $f$ and $g$ are compatible, we have $f g u=g g u=$ $f f u=g f u$.

Finally, using $\left(C_{2}\right)$, we get

$$
d(g u, g g u) \leq d^{\frac{1}{q}}(f u, f g u) \leq d^{\frac{1}{q}}(g u, g g u),
$$

that is, $g u=g g u$. We also have $g u=g g u=f g u$ and hence $g u$ is a common fixed point of $f$ and $g$.

Now, suppose that $f$ and $g$ are $R$-weakly commuting of type $\left(A_{f}\right)$. Now the weak reciprocal continuity of $f$ and $g$ implies that $\lim _{n \rightarrow \infty} f g x_{n}=f z$ or $\lim _{n \rightarrow \infty} g f x_{n}=g z$.

Let $\lim _{n \rightarrow \infty} f g x_{n}=f z$. Then the $R$-weak commutativity of type $\left(A_{f}\right)$ of $f$ and $g$ implies

$$
d\left(g g x_{n}, f g x_{n}\right) \leq d^{R}\left(f x_{n}, g x_{n}\right)
$$

Letting $n \rightarrow \infty$, we have

$$
\lim _{n \rightarrow \infty} d\left(g g x_{n}, f z\right) \leq d^{R}(z, z)=1 .
$$

This proves $\lim _{n \rightarrow \infty} g g x_{n}=f z$. Again, using $\left(C_{2}\right)$, we get

$$
d\left(g z, g g x_{n}\right) \leq d^{\frac{1}{q}}\left(f z, f g x_{n}\right)
$$

Letting $n \rightarrow \infty$, we get

$$
d(g z, f z) \leq d^{\frac{1}{q}}(f z, f z)=1
$$


Hence, we get $f z=g z$. Again, by using the $R$-weak commutativity of type $\left(A_{f}\right)$, we have

$$
d(g g z, f g z) \leq d^{R}(g z, f z)=d^{R}(f z, f z)=1,
$$

which implies that $g g z=f g z$. Therefore $f f z=f g z=g f z=g g z$. Using $\left(C_{2}\right)$, we get

$$
d(g z, g g z) \leq d^{\frac{1}{q}}(f z, f g z)=d^{\frac{1}{q}}(g z, g g z),
$$

that is, $g z=g g z$. Then we have $g z=f g z=g g z$ and hence $g z$ is a common fixed point of $f$ and $g$.

Similarly, if we assume $\lim _{n \rightarrow \infty} g f x_{n}=g z$, we can get the same result.

Now suppose that $f$ and $g$ are $R$-weakly commuting of type $\left(A_{g}\right)$. Again, similarly we have $f z$ is a common fixed point of $f$ and $g$.

Finally suppose that $f$ and $g$ are $R$-weakly commuting of type $(P)$. Since $f$ and $g$ are weakly reciprocally continuous, we have $\lim _{n \rightarrow \infty} f g x_{n}=f z$ or $\lim _{n \rightarrow \infty} g f x_{n}=$ $g z$. that

Let $\lim _{n \rightarrow \infty} f g x_{n}=f z$. Then $R$-weakly commutativity of type $(P)$ implies

$$
d\left(f f x_{n}, g g x_{n}\right) \leq d^{R}\left(f x_{n}, g x_{n}\right) .
$$

Letting $n \rightarrow \infty$, we have

$$
d\left(f f x_{n}, g g x_{n}\right) \leq d^{R}(z, z)=1,
$$

that is, $\lim _{n \rightarrow \infty} d\left(f f x_{n}, g g x_{n}\right)=1$. Also we have $f g x_{n-1}=f f x_{n} \rightarrow f z$ as $n \rightarrow \infty$ implies $g g x_{n} \rightarrow f z$ as $n \rightarrow \infty$. Also using $\left(C_{2}\right)$, we have

$$
d\left(g z, g g x_{n}\right) \leq d^{\frac{1}{q}}\left(f z, f g x_{n}\right)
$$

Letting $n \rightarrow \infty$, we get

$$
d(g z, f z) \leq d^{\frac{1}{q}}(f z, f z)=1
$$

Hence, $f z=g z$. Now using $R$-weak commutativity of type $(P)$,

$$
d(f f z, g g z) \leq d^{R}(f z, g z)=1 .
$$

This implies that $f f z=g g z$. Therefore, $f f z=f g z=g f z=g g z$. Using $\left(C_{2}\right)$, we get

$$
d(g z, g g z) \leq d^{\frac{1}{q}}(f z, f g z)=d^{\frac{1}{q}}(g z, g g z) .
$$


This implies that $g z=g g z$. Hence $g z=g g z=f g z$ and $g z$ is a common fixed point of $f$ and $g$.

Similarly, if we assume $\lim _{n \rightarrow \infty} g f x_{n}=g z$, we can get the same result.

Uniqueness follows easily in all the cases.

Example 3.5. Let $X=\mathbb{R}_{+}$with the usual multiplicative metric $d$. Define $f$ and $g: X \rightarrow X$ by $f x=x^{3}$ and $g x=x$ for all $x \in X$. Then $d(f g x, g g x)=\left|x^{2}\right|$ and $d(f x, g x)=\left|x^{2}\right|$. Then clearly $d(f g x, g g x) \leq d^{R}(f x, g x)$ for $R>1>0$ and hence $f$ and $g$ are $R$-weakly commuting of type $\left(A_{f}\right)$. Also $f$ and $g$ satisfy the condition

$$
d(f x, f y) \geq d^{q}(g x, g y)
$$

for all $x, y \in X$, where $1<q<3$. Clearly $f$ is continuous and $g(X)=f(X)=$ $X$, that is, $g(X) \subset f(X)$. We also note that $f$ and $g$ are weakly reciprocally continuous. All the conditions of Theorem 3.4 hold. Hence $f$ and $g$ have a unique common fixed point 1.

\section{References}

[1] M. Abbas, B. Ali, Y.I. Suleiman, Common fixed points of locally contractive mappings in multiplicative metric spaces with application, Int. J. Math. Math. Sci., 2015 (2015), Article ID 218683, 7 pages. doi: 10.1155/2015/218683.

[2] A.E. Bashirov, E.M. Kurplnara, A. Ozyapici, Multiplicative calculus and its applications, J. Math. Anal. Appl., 337 (2008), 36-48. doi: 10.1016/j.jmaa.2007.03.081

[3] X. He, M. Song, D. Chen, Common fixed points for weak commutative mappings on a multiplicative metric space, Fixed Point Theory Appl., 48 (2014), 9 pages. doi: $10.1186 / 1687-1812-2014-48$

[4] S.M. Kang, P. Kumar, S. Kumar, P. Nagpal, S.K. Garg, Common fixed points for compatible mappings and its variants in multiplicative metric sapces, Int. J. Pure Appl. Math. 102 (2015), 383-406.

[5] S. Kumar, S.K. Garg, Expansion mapping theorems in metric spaces, Int. J. Contemp. Math. Sci. 4(2009), 1749-1758.

[6] M. Özavsar, A.C. Çevikel, Fixed points of multiplicative contraction mappings on multiplicative metric spaces, arXiv:1205.5131v1 [math.GM], 2012, 14 pages.

[7] R.P. Pant, Common fixed points of noncommuting mappings, J. Math. Anal. Appl. 188 (1994), 436-440. doi: 10.1006/jmaa.1994.1437

[8] R.P. Pant, Common fixed points of four mappings, Bull. Calcutta math. Soc. 90 (1998), 281-286.

[9] R.P. Pant, Common fixed point theorem under a new condition, Indian J. Pure Appl. Math. 30 (1999), 147-152.

[10] H. K. Pathak, Y.J. Cho, S.M. Kang, Remarks of R-weakly commuting and common fixed point theorems, Bull. Korean Math. Soc. 34 (1997), 247-257. 
[11] B.E. Rhoades, An expansion mapping theorem, Jñānābha, 23 (1993), 151-152.

[12] M. Sarwar, R. Badshah-e, Some unique fixed point theorems in multiplicative metric space, arXiv:1410.3384v2 [math.GM], 2014, 19 pages.

[13] S.Z. Wang, B.Y, Li, Z.M. Gao, K. Iséki, Some fixed point theorems on expansion mappings, Math. Japon., 29 (1984), 631-636. 